\title{
ON THE RANGE KERNEL ORTHOGONALITY AND P-SYMMETRIC OPERATORS
}

\author{
SAID BOUALI AND YOUSSEF BOUHAFSI
}

\begin{abstract}
Let $H$ be a separable infinite dimensional complex Hilbert space, and let $L(H)$ denote the algebra of all bounded linear operators on $H$. For given $A \in L(H)$, we define the derivation $\delta_{A}: L(H) \longrightarrow L(H)$ by $\delta_{A}(X)=A X-X A$. In this paper we establish the orthogonality of the range $R\left(\delta_{A}\right)$ and the kernel $\operatorname{ker}\left(\delta_{A}\right)$ of a derivation $\delta_{A}$ induced by a cyclic subnormal operator $A$, in the usual sense. We give a version of the Putnam - Fuglede theorem. We establish a short proof of the principal result of F. Wenying and J. Guoxing in [10]. Relatad results for P-symmetric operators are also given.
\end{abstract}

Mathematics subject classification (2000): 47B47, 47B10, 47A30.

Key words and phrases: Range-kernel orthogonality, subnormal operator, cyclic vector, Putnam Fuglede theorem, P-symmetric operator.

\section{REFERENCES}

[1] J. H. Anderson, On normal derivations, Proc. Amer. Math. Soc., 38, (1973), 135-140.

[2] J. H. Anderson, J. W. Bunce, J. A. Deddens And J. P. Williams, $C^{*}$-algebras and derivations ranges, Acta. Sci. Math., 40, (1978), 211-227.

[3] M. BENLARBI, S. BOUALI AND S. CHERKI, Une remarque sur l'orthogonalité de l'image au noyau d'une dérivation généralisée, Proc. Amer. Math. Soc., 126, (1998), 167-171.

[4] C. A. BERGER, B. I. SHAW, Self-commutator of multicyclic hyponormal operators are always trace class, Bull. Amer. Math. Soc., 79, (1973), 1193-1199.

[5] S. Bouali, J. Charles, Extension de la notion d'opérateur d-symétrique I, Acta. Sci. Math., 58, (1993), $517-525$.

[6] S. Bouali, J. Charles, Extension de la notion d'opérateur d-symétrique II, Linear algebra and its applications, 225, (1995), 175-185.

[7] J. B. ConwaY, A course in functional analysis, Springer Verlag, New York, Berlin, Heidelberg ,(1990).

[8] B. P. DugGal, On intertwining operators, Monatsh Math., 106, (1988), 139-148.

[9] B. P. Duggal, A remark on normal derivations, Proc. Amer. Math. Soc., 126, (1998), 2047-2052.

[10] W. FENG, G. JI, A counter example in the theory of derivations, Glasgow Math. J., 31, (1989), 161-163.

[11] P. A. Fillmore, J. G. Stampfli, AND J. P. Williams, On the essential numerical runge, the essential spectrum, and a problem of Halmos, Acta Sci. Math., 33, (1972), 179-192.

[12] I. C. GohberG, M. G. KreIN, Introduction to the theory of linear nonself adjoint operators, transl. Math. Monographs, 18, A. Math. Soc., Providence, R. I. (1969).

[13] D. A. HERRERO, Approximation of Hilbert space operators I, Pitman Advenced Publishing Program, Boston-London-Milbourne (1982).

[14] F. KitTANEH, On normal derivations of Hilbert-Schmidt type, Glasgow Math. J., 29, (1987), 245-248.

[15] F. Kittaneh, Normal derivations in norm ideals, Proc. Amer. Math. Soc., 123, (1995), 1779-1785.

[16] F. KitTaneH, Operators that are orthogonal to the range of a derivation, J. Math. Anal. Appl., 203, (1997), 868-873.

[17] P. J. MAHER, Commutator Approximants, Proc. Amer. Math. Soc., 115, (1992), 995-1000.

[18] R. L. Moore, D. D. Rogers, Note on intertwining M-hyponormal operators, Proc. Amer. Math. Soc., 83, (1981), 514-516.

[19] J. P. WiLliams, Finite operators, Proc. Amer. Math. Soc., 26, (1970), 129-136.

[20] T. Yoshino, Subnormal operators with a cyclic vector, Tôhoku Math. J., 21, (1969), 47-55. 